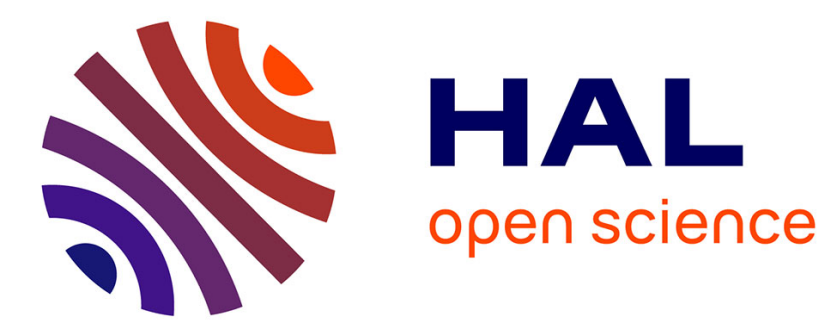

\title{
Leveraging Light Forest With Rateless Network Coding to Design Efficient All-Optical Multicast Schemes for Elastic Optical Networks
}

Lulu Yang, Long Gong, Fen Zhou, Bernard Cousin, Miklós Molnár, Zuqing Zhu

\section{To cite this version:}

Lulu Yang, Long Gong, Fen Zhou, Bernard Cousin, Miklós Molnár, et al.. Leveraging Light Forest With Rateless Network Coding to Design Efficient All-Optical Multicast Schemes for Elastic Optical Networks. Journal of Lightwave Technology, 2015, 33 (18), pp.3945-3955. 10.1109/JLT.2015.2457092 . hal-01216710

\author{
HAL Id: hal-01216710 \\ https://hal.science/hal-01216710
}

Submitted on 16 Oct 2015

HAL is a multi-disciplinary open access archive for the deposit and dissemination of scientific research documents, whether they are published or not. The documents may come from teaching and research institutions in France or abroad, or from public or private research centers.
L'archive ouverte pluridisciplinaire $\mathbf{H A L}$, est destinée au dépôt et à la diffusion de documents scientifiques de niveau recherche, publiés ou non, émanant des établissements d'enseignement et de recherche français ou étrangers, des laboratoires publics ou privés. 


\title{
Leveraging Light-Forest with Rateless Network Coding to Design Efficient All-Optical Multicast Schemes for Elastic Optical Networks
}

\author{
Lulu Yang, Long Gong, Fen Zhou, Bernard Cousin, Miklós Molnár, and Zuqing Zhu, Senior Member, IEEE
}

\begin{abstract}
In this paper, we study the multicast-capable routing, modulation and spectrum assignment (MC-RMSA) schemes that consider the physical impairments from both the transmission and light-splitting in elastic optical networks (EONs). Specifically, we propose to provision each multicast request with a light-forest which consists of one or more light-trees to avoid the dilemma that because of the accumulated physical impairments, a relatively large light-tree may have to use the lowest modulationlevel and hence consume too many frequency slots (FS'). In order to further improve the spectral efficiency and compensate for the differential delays among the light-trees, we incorporate the rateless network coding (R-NC) in the multicast system. We first formulate an integer linear programming (ILP) model to solve the problem for static network planning. Then, we propose three time-efficient heuristics that leverage the set-cover problem and utilize layered auxiliary graphs. The simulation results indicate that in both the ILP and heuristics, the MC-RMSA with R-NC can achieve better performance on the maximum index of used FS' than that without. After that, we evaluate the heuristics in dynamic network provisioning. The results show that the MCRMSA with R-NC can effectively improve the performance of all-optical multicast in EONs to reduce the blocking probability.
\end{abstract}

Index Terms-All-optical multicast; Routing, modulation and spectrum assignment (RMSA); Light-forest; Rateless network coding; Elastic optical networks (EONs).

\section{INTRODUCTION}

O VER the past decade, the rapid development of bandwidth intensive applications has made the traffic volumes in Internet backbone increase exponentially. To address this issue, we need to realize highly flexible and scalable backbone networks, which has stimulated active research and development on new optical networking technologies. In line of these efforts, people have developed advanced optical transmission and switching technologies $[1,2]$, and used them to realize the flexible-grid elastic optical networks (EONs) [3, 4]. It is known that with the bandwidth-variable transponders (BVTs) and wavelength-selective switches (BV-WSS'), EONs can achieve the bandwidth allocation granularity at $12.5 \mathrm{GHz}$ or less and support a super-channel at $400 \mathrm{GHz}$ and beyond

L. Yang, L. Gong, and Z. Zhu are with the School of Information Science and Technology, University of Science and Technology of China, Hefei, Anhui 230027, P. R. China (email: zqzhu@ieee.org).

F. Zhou is with LIA lab of the University of Avignon, France (email: fen.zhou@univ-avignon.fr).

B. Cousin is with the IRISA lab of the University of Rennes, France (email: bernard.cousin@irisa.fr).

M. Molnár is with the LIRMM lab of the University of Montpellier, France (email: molnar@lirmm.fr).

Manuscript received on Dec. 5, 2014 as well. Therefore, compared with the traditional fixed-grid wavelength-division multiplexing (WDM) networks, EONs provide enhanced spectral efficiency and make the spectrum allocation in the optical layer more flexible.

Besides these advantages, EONs also bring new challenges to the network control and management (NC\&M), since the elastic nature determines that the network planning and provisioning procedure would be more sophisticated than its counterpart in conventional WDM networks. Specifically, to establish a lightpath in an EON, the network operator needs to allocate a few spectrally-contiguous frequency slots (FS') to satisfy the bandwidth demand. Here, the bandwidth of an FS is usually at $12.5 \mathrm{GHz}$, which is much narrower than a wavelength channel. Moreover, the modulation format used by the FS' should be chosen adaptively from those that have different spectral efficiencies and receiver sensitivities, e.g., binary phase-shifted keying (BPSK), quadrature phaseshifted keying (QPSK), 8 quadrature amplitude modulation (8QAM) and 16-QAM, according to the quality-of-transmission (QoT). Intuitively, if we change the modulation format to a higher order one, e.g., from QPSK to 8-QAM, the spectral efficiency becomes higher and thus we can use fewer FS' to provision the same bandwidth demand. Meanwhile, since the receiver sensitivity of 8-QAM is lower, it can only support a shorter transmission reach. To this end, the classic routing and wavelength assignment (RWA) problem in WDM networks evolves into the routing, modulation and spectrum assignment (RMSA) problem in EONs [5, 6].

Previously, numerous studies have addressed the RMSA problem and proposed various approaches to solve it with different optimization objectives [3, 5-11]. Nevertheless, most of them did not consider the all-optical multicasting that can realize point-to-multiple-point communications in EONs. With the evolution of the Internet, multicast has become a key and necessary communication scheme to efficiently support emerging network services such as grid computing and teleconferencing, etc. Moreover, with the recent rise of interdatacenter networks, huge-throughput traffics for data backup or service migration may also require multicast transmission. Hence, it is also desired to facilitate efficient multicast schemes in the backbone networks. All-optical multicast with light-trees has been proposed in [12] for the IP-over-WDM networks. Basically, by leveraging the multicast-capable optical crossconnects (MC-OXCs) [13, 14], all-optical multicast allows the nodes on a light-tree to send the optical signal to more than one outputs (i.e., light-splitting), and reduces the cost from 
optical-to-electrical-to-optical (O/E/O) conversions [15].

The RWA problem for all-optical multicast in WDM networks has been studied in $[12,15-20]$. Due to the unique requirements on NC\&M (e.g., RMSA for resource allocation), supporting efficient all-optical multicast in EONs would be more challenging and has just started to attract research interests since recently. The authors of [21] first studied alloptical multicast in EONs and compared the performance of two simple multicast-capable routing and spectrum assignment (MC-RSA) algorithms. However, they did not consider either the QoT constraint or the adaptive modulation selection. In [22], we designed an approach to facilitate MC-RSA with layered auxiliary graphs and demonstrated that it could outperform those in [21]. Nevertheless, the QoT-aware modulation selection was still not addressed. By using an over-simplified impairment model that did not consider the optical signalto-noise-ratio (OSNR) degradation due to light-splitting, we studied the RMSA for all-optical multicast (MC-RMSA) in EONs, formulated two integer linear programming (ILP) models, and proposed several heuristics based on genetic algorithm in [23]. However, it is known that the light-splitting in MCOXCs causes power loss and the subsequent re-amplification results in noticeable OSNR degradation $[15,18]$. Hence, one cannot simply assume that the transmission reaches of the optical signals with and without light-splitting are the same.

In this work, we investigate the MC-RMSA schemes that consider the physical impairments from both the transmission and light-splitting in EONs. Specifically, we propose to serve each multicast request with a light-forest that consists of one or more light-trees to avoid the situation that due to the accumulated impairments, a relatively large light-tree may have to use the lowest modulation-level and hence consume too many FS'. Moreover, to further improve the spectral efficiency and compensate for the latency differences among the light-trees, we propose to use the rateless network coding (R-NC) in [24] in the multicast system. We first formulate an ILP model to tackle the problem of static network planning and obtain the optimal solutions of small-scale problems. Then, we leverage the set-cover problem and layered auxiliary graphs to design time-efficient heuristics, and use them for dynamic network provisioning. The proposed algorithms are evaluated with extensive simulations, and the results show that the MCRMSA using light-forest with R-NC can effectively improve the performance of all-optical multicast in EONs.

The rest of the paper is organized as follows. Section II introduces the network model and explains how to use the light-forest with R-NC to realize all-optical multicast in EONs. In Section III, we formulate the ILP model to jointly optimize the light-forest construction, modulation format selection, and spectrum assignment (i.e., MC-RMSA) for multicast requests. The heuristics for MC-RMSA are proposed in Section IV, and Section V discusses the numerical simulations for performance evaluation. Finally, Section VI summarizes the paper.

\section{Problem Description}

\section{A. Network Model}

We use a directed graph $G(V, E)$ to represent the EON's physical topology, where $V$ denotes the set of nodes that each

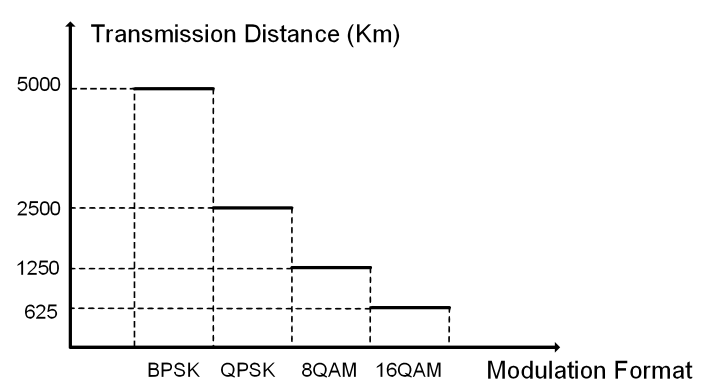

Fig. 1. Mapping between transmission distance and modulation format.

equips with an MC-OXC, and $E$ is the fiber link set. Each link $e \in E$ has a bandwidth capacity of $\digamma$ FS', each of which occupies a fixed bandwidth and provides a capacity of $C \mathrm{~Gb} / \mathrm{s}$ when using BPSK as the modulation format. For the modulation format selection, we define $m$ as the modulationlevel, and have $m=1,2,3$, and 4 for BPSK, QPSK, 8-QAM, and 16-QAM, respectively. Hence, for different modulation formats, the capacity of an FS can be calculated as $m \cdot C \mathrm{~Gb} / \mathrm{s}$. Here, since we consider all-optical multicast without spectrum conversions, the modulation format and spectrum assignment stay unchanged for all the links on a light-tree.

For a light-tree, the modulation-level is selected according to its QoT, which depends on both the transmission distance of the longest branch and the number of destinations (i.e., lightsplitting times) [15]. We first set up the mapping between the modulation-level and the maximum transmission distance for the cases in which there is no light-splitting (i.e., unicast). Fig. 1 depicts the mapping, which is obtained based on the experimental results in [25]. With this mapping, we always select the highest feasible modulation-level to use as long as the transmission distance permits, for obtaining the highest spectral efficiency $[5,6]$. Then, we address the additional impairments due to the light-splitting in MC-OXCs.

Definition The relation among the modulation-level, the transmission distance and the number of destinations in a lighttree is referred as the MTD relation. For a light-tree that includes $n$ destinations, the transmission distance of its longest branch and the modulation-level $m$ to be used should satisfy the equation below $[15,18]$

$$
S_{m, n}=\frac{S_{m, 1}}{\log _{10}(n)+1}
$$

where $S_{m, n}$ denotes the maximum length that the light-tree's longest branch can have to use modulation-level $m$, and $S_{m, 1}$ follows the mapping in Fig. 1.

A multicast request can be denoted as $M R(s, D, B)$, where $s \in V$ is the source node, $D \subseteq V \backslash s$ represents the set of destination nodes, and $B$ is the capacity requirement in $\mathrm{Gb} / \mathrm{s}$. Due to the MTD relation, when the size of $D$ is relatively large and/or the distances between $s$ and $D$ are long, it would be impossible or inefficient to serve $M R$ with a single lighttree. Therefore, we have to consider MC-RMSA with lightforest, and the construction of the light-forest and the selection of modulation-levels for the light-trees in it are correlated. 
With Eq. (1), we determine the modulation-level $m_{k}$ for the $k$-th light-tree in the light-forest, and assign $\left\lceil\frac{B}{m_{k} \cdot C}\right\rceil$ spectrallycontiguous FS' on the links in the light-tree.

\section{B. Multicast using Light-Forest with R-NC}

Note that in addition to accommodating the QoT constraint, we may also use a light-forest to serve $M R$ because a large FS-block ${ }^{1}$ cannot be found on the links due to spectrum fragmentation [26]. Hence, when building the light-forest, we may not only divide the destinations in $D$ into groups and cover each with a light-tree, but also split the traffic to certain destination(s) into multiple sub-streams and send them over several light-trees. The latter mimics the spectrum-splitting scheme for serving unicast lightpaths [11, 27].

However, the aforementioned MC-RMSA with light-forest has some intrinsic drawbacks. First of all, the total spectrum usage may become higher. Basically, we can easily prove that when a light-tree and a light-forest that consists of multiple light-trees are both feasible for $M R$, the total number of links in the light-forest is equal to or larger than that in the lighttree $^{2}$. Therefore, if we cannot leverage the adaptive modulation selection to reduce the spectrum usage on each link, the lightforest may consume more spectra. Secondly, if we consider the case that the traffic to a certain destination is split into multiple sub-streams, the differential delay among the substreams may incur a relatively large buffer at the receiver for data reordering [28].

In order to relieve the impacts from these drawbacks, we propose to incorporate the rateless network coding (R-NC) [24] in the multicast system. Specifically, the work in [24] indicated that with $\mathrm{R}-\mathrm{NC}$, we can recover $k$ original symbols by using any $k \cdot(1+\varepsilon)$ encoded symbols, where the order to receive the encoded symbols does not affect the decoding results. Here, $\varepsilon$ is a small real number that usually satisfies $\varepsilon \leq 0.05$ [29]. Therefore, we can see that all-optical multicast using light-forest with R-NC is promising. Actually, previous studies have already considered the usage of R-NC for the multicast in multimedia networks [29, 30].

We use Figs. 2-4 as intuitive examples to explain the working principle and benefits of multicast using light-forest with R-NC. Fig. 2 shows the spectrum resources on each link in the network. The source node is $s$, and the destination nodes are $D=\left\{d_{1}, d_{2}, d_{3}\right\}$. To simplify the problem, we do not consider the adaptive modulation selection, and assume that if the longest branch of a light-tree is more than two hops, only one destination can be reached. Here, the capacity of an FS is $12.5 \mathrm{~Gb} / \mathrm{s}$, and each destination node needs a bandwidth of $20 \mathrm{~Gb} / \mathrm{s}$, which means it needs $\left\lceil\frac{20}{12.5}\right\rceil=2$ contiguous FS'. However, we cannot find two available contiguous FS' in any light-tree to satisfy the requirement. Hence, we try to split the traffic over multiple light-trees to serve the request.

Fig. 3 considers the case without R-NC. We simply split the traffic into two sub-streams, i.e., $A$ and $B$, each of which

\footnotetext{
${ }^{1}$ An FS-block is the block of available contiguous FS' in the optical spectrum, which has the maximum size in FS' at the spectral location.

${ }^{2}$ Here, if more than one light-tree in the light-forest use the same link, we count the link multiple times since the source will deliver multiple copies of the traffic over it.
}

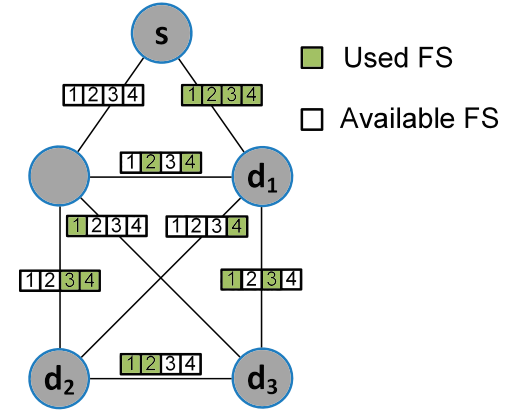

Fig. 2. Spectrum resources on links in an EON.

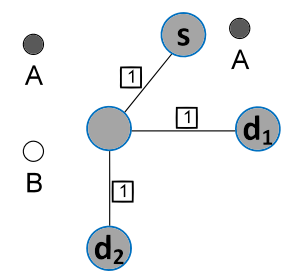

(a)

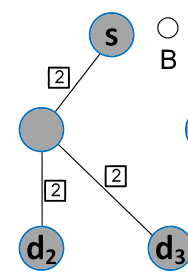

(b)

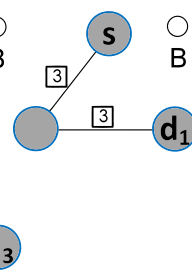

(c)

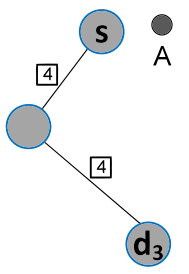

(d)
Fig. 3. Example on multicast using light-forest without R-NC.

carries a bandwidth of $10 \mathrm{~Gb} / \mathrm{s}$ (i.e., $1 \mathrm{FS}$ ), and build a lightforest with 4 light-trees to deliver them. In Fig. 3, we can find that it is not possible to merge the light-trees in Figs. 3(c) and 3 (d), even though they can use FS 3 from $s$ to $d_{1}$ and $d_{3}$. This is because if we do so, neither $d_{1}$ nor $d_{3}$ can receive both $A$ and $B$. In this case, the multicast scheme in Fig. 3 consumes a total bandwidth resource of 10 FS.hops. Moreover, we will have the data reordering issue if the light-trees' branches are in different lengths. Fig. 4 shows the case with R-NC. Here, we use R-NC to encode the traffic into three sub-streams $a$, $b$, and $c$, each of which requires a bandwidth of $10 \cdot(1+\varepsilon)$ $\mathrm{Gb} / \mathrm{s}$. Then, according to the working principle of R-NC, we can just build 3 light-trees to ensure that all the destinations can receive $20 \cdot(1+\varepsilon) \mathrm{Gb} / \mathrm{s}$ of encoded bandwidth for correct decoding. For instance, we can deliver sub-streams $a, b$, and $c$ over the light-trees as shown in Fig. 4(a)-(c). Then, for this case, the total bandwidth consumption is $9 \mathrm{FS} \cdot \mathrm{hops}$, and we do not have to worry about the data reordering issue.

With this network model and considering the advantages of R-NC, we study MC-RMSA for two different scenarios of EONs, i.e., static network planning and dynamic network pro-

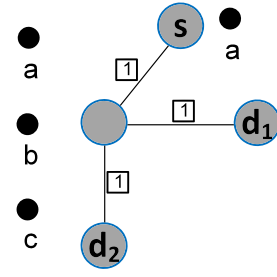

(a)

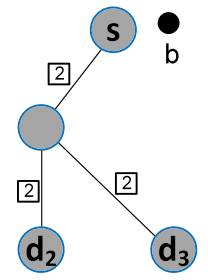

(b)

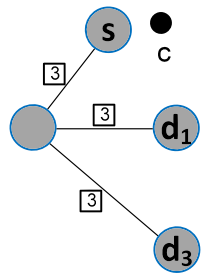

(c)
Fig. 4. Example on multicast using light-forest with R-NC. 


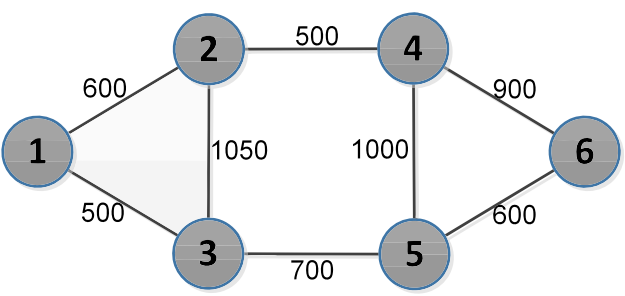

Fig. 5. Six-node topology marked with link lengths in kilometers.

visioning. In the static network planning, we try to minimize the maximum index of used FS' and make the MC-RMSA more spectral efficient, while in the dynamic network provisioning, the request blocking probability should be minimized to make the MC-RMSA more effective.

\section{ILP FORMULATION}

In this section, we formulate an ILP model to optimize MC-RMSA for multicast requests. Specifically, we consider the light-forest construction, modulation format selection and spectrum assignment jointly, and incorporate the R-NC scheme discussed in the previous section.

\section{Parameters:}

- $G(V, E)$ : Network topology, where $V$ and $E$ are the sets of nodes and fiber links, respectively.

- $l_{(u, v)}$ : Length of link $(u, v) \in E$ in kilometers.

- $C$ : Capacity of an FS in Gb/s when using BPSK as the modulation format.

- $s$ : Source node of the multicast request $M R$.

- $D$ : Destination set of $M R$ and each destination is $d \in D$.

- $F$ : Number of FS' on each link.

- B: Capacity requirement of $M R$ in $\mathrm{Gb} / \mathrm{s}$.

- $B_{g}$ : Minimum capacity that can be allocated on a lighttree when we use R-NC, in terms of $\mathrm{Gb} / \mathrm{s}$.

- $[K]:[K]=\{1,2, \ldots, K\}, K$ is the maximum number of light-trees that can be included in the light-forest ${ }^{3}$. The index of a light-tree is $k \in[K]$.

- $Q: Q=\left\lceil\frac{B}{B_{g}}\right\rceil$, is the maximum value of $B$ in terms of $B_{g}$, and the index $q$ satisfies $q \in[Q]$.

- $\Delta$ : Maximum transmission reach when using BPSK with single destination, i.e., $\Delta=S_{1,1}$ based on Eq. (1).

- $M$ : Highest modulation-level.

- $\mathfrak{T}_{(u, v)}$ : Number of available FS-blocks on link (u, v).

- $W_{(u, v), t}$ : Start-index of the $t$-th available FS-block on link $(u, v)$.

- $Z_{(u, v), t}$ : End-index of the $t$-th available FS-block on link $(u, v)$.

- $S_{m, n}$ : MTD relation, which tells the maximum transmission reach of a branch when there are $n$ destinations in a light-tree that uses modulation-level $m$.

\section{Variables:}

- $f_{d, k}^{(u, v)}$ : Boolean variable that indicates whether link $(u, v)$ is used to serve destination $d \in D$ in the $k$-th light-tree.

- $F_{k}^{(u, v)}$ : Boolean variable that equals 1 if link $(u, v)$ is in the $k$-th light-tree, and 0 otherwise.

\footnotetext{
${ }^{3}$ Note that when we split the traffic into multiple sub-streams, we count a light-tree multiple times if it carries more than one sub-stream.
}

- $y_{d, k}$ : Boolean variable that indicates whether destination $d \in D$ gets traffic from the $k$-th light-tree.

- $x_{k}$ : Integer variable that represents the number of destinations covered by the $k$-th light-tree.

- $\Delta_{k}$ : Integer variable that represents the length of the longest branch on the $k$-th light-tree.

- $m_{k}$ : Integer variable that represents the modulation-level used on the $k$-th light-tree.

- $o_{k_{1}, k_{2}}$ : Boolean variable that equals 1 , if the start-index of the FS-block used on the $k_{1}$-th light-tree is smaller than that of the $k_{2}$-th light-tree, and 0 otherwise.

- $c_{k_{1}, k_{2}}$ : Boolean variable that equals 1 , if the $k_{1}$-th and $k_{2}$-th light-trees share common link(s), and 0 otherwise.

- $w_{k}$ : Integer variable that represents the start-index of the FS-block used on the $k$-th light-tree.

- $z_{k}$ : Integer variable that represents the end-index of the FS-block used on the $k$-th light-tree.

- $\gamma_{k}^{m, n}$ : Boolean variable that indicates whether the $k$-th light-tree satisfies the specific $m$ and $n$ according to the MTD relation, where $m \in[M]$ is the modulation-level and $n$ is the number of destinations.

- $\xi_{k}$ : Integer variable that represents the capacity allocated to the $k$-th light-tree in terms of $B_{g}$.

- $\rho_{d, k}^{q, 0}$ : Boolean variable that equals 1 if the capacity allocated to the $k$-th light-tree is $q \cdot B_{g}$ and the $k$-th lighttree does not cover $d$, and 0 otherwise.

- $\rho_{d, k}^{q, 1}$ : Boolean variable that equals 1 if the capacity allocated to the $k$-th light-tree is $q \cdot B_{g}$ and the $k$-th lighttree covers $d$, and 0 otherwise.

- $h_{k}^{q, m}$ : Boolean variable that indicates whether on the $k$ th light-tree, the capacity allocated is $q \cdot B_{g}$ and the modulation-level chosen is $m$.

- $h_{k}^{m}$ : Boolean variable that indicates whether on the $k$-th light-tree, the modulation-level chosen is $m$.

- $\Omega$ : Maximum index of the used FS' in the light-forest.

- $u_{k}^{(u, v), t}$ : Boolean variable that equals 1 , if the $t$-th available FS-block on link $(u, v)$ is assigned to the $k$-th lighttree, and 0 otherwise.

\section{Objective:}

We design a metric as follows to assist the optimization.

$$
\Psi=\alpha_{1} \cdot \Omega+\alpha_{2} \cdot \sum_{(u, v) \in E} \sum_{k \in[K]} F_{k}^{(u, v)}+\sum_{k \in[K]} \frac{\Delta_{k}}{\Delta},
$$

where $\alpha_{1}$ and $\alpha_{2}$ are positive constants $\left(\alpha_{1} \gg \alpha_{2}\right)$ to balance the ratio among the three terms in Eq. (2). The first term is for the maximum index of the used FS' for the request (i.e., $\Omega$ ), and a smaller $\Omega$ reflects a more efficient MC-RMSA, as we can make the spectrum utilization more compact in the network. Therefore, we use $\alpha_{1}$ to make sure that this term makes the major contribution to $\Psi$. The second term is the total number of used links in the light-forest, which is less important than $\Omega$, but is also needed to assist the optimization. We use the third term to ensure that each tree has the minimum $\Delta_{k}$. The last two terms are necessary because the ILP only provides the MC-RMSA for one multicast request, and when there are multiple pending requests, we will use the ILP repeatedly to find the MC-RMSAs for them one by one. 
Hence, to minimize the values of these two variables for the current request can benefit the rest requests. With Eq. (2), we define the optimization objective as

$$
\text { Minimize } \Psi \text {. }
$$

\section{Constraints:}

1) Flow Conservation Constraint,

$$
\begin{aligned}
\sum_{u \in V} f_{d, k}^{(u, v)}-\sum_{u \in V} f_{d, k}^{(v, u)} & = \\
& \left\{\begin{array}{rl}
-y_{d, k}, \quad v & =s, \\
y_{d, k}, \quad v & =d, \\
0, \quad \text { otherwise, }
\end{array} \quad \forall d \in D, k \in[K] .\right.
\end{aligned}
$$

Eq. (4) ensures that if a light-tree covers a destination node $d$, there is a single path from $s$ to $d$ on it.

2) Link Aggregation Constraint,

$$
F_{k}^{(u, v)} \geq f_{d, k}^{(u, v)}, \quad \forall k \in[K], d \in D .
$$

Eq. (5) ensures that if different destinations share the same link(s) in a light-tree, we aggregate the links into one.

3) Impairment-Related Constraints,

$$
\begin{gathered}
\sum_{(u, v) \in E} f_{d, k}^{(u, v)} \cdot l_{(u, v)} \leq \Delta_{k}, \quad \forall k \in[K], d \in D, \\
\Delta_{k} \leq \Delta, \quad \forall k \in[K] .
\end{gathered}
$$

Eqs. (6) - (7) ensure that the lengths of the branches in each light-tree should not be longer than that of the longest branch in the light-tree or the maximum transmission reach determined by the MTD relation.

$$
\begin{gathered}
x_{k}=\sum_{d \in D} y_{d, k}, \quad \forall k \in[K], \\
m_{k}=\left\lfloor\log _{2}\left(\frac{\Delta}{\Delta_{k}}\right)-\log _{2}\left[\log _{10}\left(x_{k}\right)+1\right]\right\rfloor+1, \quad \forall k \in[K] \\
m_{k} \leq M, \quad \forall k \in[K] .
\end{gathered}
$$

Eqs. (8) - (10) determine the modulation-level chosen for the $k$-th light-tree. Since the expression in Eq. (9) is nonlinear, we introduce the variable $\gamma_{k}^{m, n}$ to linearize it and transform the constraint in Eq. (9) into a set of equations as

$$
\begin{gathered}
\sum_{m} \sum_{n} \gamma_{k}^{m, n} \leq 1, \quad \forall k \in[K], \\
\sum_{m} \sum_{n} n \cdot \gamma_{k}^{m, n}=x_{k}, \quad \forall k \in[K], \\
\sum_{m} \sum_{n} S_{m, n} \cdot \gamma_{k}^{m, n} \geq \Delta_{k}, \quad \forall k \in[K], \\
m_{k} \leq \sum_{m} \gamma_{k}^{m, n} \cdot m, \quad \forall k \in[K] .
\end{gathered}
$$

4) Spectrum Assignment Constraints,

$$
\sum_{t \in\left[\mathfrak{T}_{(u, v)}\right]} u_{k}^{(u, v), t}=F_{k}^{(u, v)}, \quad \forall k \in[K]
$$

$$
w_{k} \geq u_{k}^{(u, v), t} \cdot W_{(u, v), t}, \quad \forall k \in[K],(u, v) \in E, t \in\left[\mathfrak{T}_{(u, v)}\right],
$$

$$
\begin{aligned}
z_{k} \leq\left(Z_{(u, v), t}-\digamma\right) \cdot u_{k}^{(u, v), t}+\digamma & \\
& \forall k \in[K],(u, v) \in E, t \in\left[\mathfrak{T}_{(u, v)}\right] .
\end{aligned}
$$

Eqs. (15) - (17) ensure that if link $(u, v)$ is on the $k$-th lighttree, the FS' allocated to the light-tree should be located in an available FS-block on it.

$z_{k_{2}}-w_{k_{1}}+1 \leq \digamma \cdot\left(1+o_{k_{1}, k_{2}}-c_{k_{1}, k_{2}}\right), \forall k_{1}, k_{2} \in[K], k_{1} \neq k_{2}$,

$z_{k_{1}}-w_{k_{2}}+1 \leq \digamma \cdot\left(2-o_{k_{1}, k_{2}}-c_{k_{1}, k_{2}}\right), \forall k_{1}, k_{2} \in[K], k_{1} \neq k_{2}$.

Eqs. (18) - (19) ensure that for any two different light-trees sharing common link(s), their spectrum assignments can never overlap with each other.

5) Capacity Constraints,

For these constraints, we consider the cases with and without $\mathrm{R}-\mathrm{NC}$, and describe those designed for each of them.

a) Case without $R-N C$,

$$
\sum_{k \in[K]} y_{d, k} \geq 1, \quad \forall d \in D .
$$

Eq. (20) ensures that when there is no R-NC, each destination $d \in D$ only needs to be covered ${ }^{4}$ by one light-tree in the light-forest. By applying this constraint, we avoid to use traffic splitting in MC-RMSA. As discussed in Subsection II-B, traffic splitting causes several drawbacks for the case without R-NC. In the rest of the paper, we refer to the ILP model for the case without R-NC as ILP.

$$
z_{k}-w_{k}+1=\left\lceil\frac{B}{m_{k} \cdot C}\right\rceil, \quad \forall k \in[K] .
$$

Eq. (21) ensures that the number of FS' allocated to each light-tree satisfies the capacity requirement. Eq. (21) is nonlinear, and we linearize it with the following equations.

$$
\begin{gathered}
\sum_{m \in[M]} h_{k}^{m} \leq 1, \quad \forall k \in[K], \\
\sum_{m \in[M]} h_{k}^{m} \cdot m=m_{k}, \quad \forall k \in[K], \\
z_{k}-w_{k}+1 \geq \sum_{m \in[M]}\left(\frac{h_{k}^{m} \cdot B}{m \cdot C}\right), \quad \forall k \in[K] .
\end{gathered}
$$

b) Case with $R-N C$,

$$
\sum_{k \in[K]}\left(y_{d, k} \cdot \xi_{k} \cdot B_{g}\right) \geq B, \quad \forall d \in D .
$$

Eq. (25) ensures that when there is R-NC, each destination $d \in D$ receives enough encoded bandwidth to recover the

\footnotetext{
${ }^{4}$ Note that, only if $d \in D$ appears as a destination node in the light-tree, we say it is covered.
} 
original data ${ }^{5}$. In the rest of the paper, we refer to the ILP model for the case with R-NC as ILP-R-NC. Eq. (25) is nonlinear, and we introduce Eqs. (26) - (29) to linearize it.

$$
\begin{gathered}
\xi_{k}=\sum_{q \in[Q]} q \cdot\left(\rho_{d, k}^{q, 0}+\rho_{d, k}^{q, 1}\right) \cdot B_{g}, \quad \forall k \in[K], d \in D, \\
\sum_{q \in[Q]} \rho_{d, k}^{q, 1}=y_{d, k}, \quad \forall k \in[K], d \in D, \\
\sum_{q \in[Q]} \rho_{d, k}^{q, 0}=1-y_{d, k}, \quad \forall k \in[K], d \in D, \\
B_{g} \cdot\left(\sum_{k \in[K]} \sum_{q \in[Q]} \rho_{d, k}^{q, 1} \cdot q\right) \geq B, \quad \forall d \in D . \\
z_{k}-w_{k}+1=\left\lceil\frac{\xi_{k} \cdot B_{g}}{m_{k} \cdot C}\right\rceil, \quad \forall k \in[K] .
\end{gathered}
$$

Eq. (30) ensures that the number of FS' allocated to each light-tree satisfies the capacity requirement. As it is also nonlinear, we linearize it by using the following equations.

$$
\begin{gathered}
\sum_{q \in[Q]} \sum_{m \in[M]} h_{k}^{q, m} \leq 1, \quad \forall k \in[K], \\
\sum_{q \in[Q]} \sum_{m \in[M]} h_{k}^{q, m} \cdot m=m_{k}, \quad \forall k \in[K], \\
\sum_{q \in[Q]} \sum_{m \in[M]} h_{k}^{q, m} \cdot q=\xi_{k}, \quad \forall k \in[K], \\
z_{k}-w_{k}+1 \geq \sum_{q \in[Q]} \sum_{m \in[M]}\left(\frac{h_{k}^{q, m} \cdot q \cdot B_{g}}{m \cdot C}\right), \quad \forall k \in[K] .
\end{gathered}
$$

6) Common-Link-Related Constraint,

$c_{k_{1}, k_{2}} \geq F_{k_{1}}^{(u, v)}+F_{k_{2}}^{(u, v)}-1, \quad \forall k_{1} \neq k_{2} \in[K], \forall(u, v) \in E$.

Eq. (35) ensures that all the common links between any two different light-trees are taken care of.

7) Constraint on Maximum Index of Used FS' (MIUFS),

$$
\Omega \geq z_{k}, \quad \forall k \in[K] .
$$

Eq. (36) ensures that the maximum index $\Omega$ of used FS' is equal to or larger than the end-index of the FS-block used on any light-tree in the light-forest.

The variable number in the ILP is $\left(\left(|E|+M+\frac{2 \cdot B}{B_{g}}+1\right)\right.$. $\left.|D|+(|\digamma|+1) \cdot|E|+\left(\frac{B}{B_{q}}+1\right) \cdot M+6\right) \cdot K+2 \cdot K^{2}+1$, and the constraint number is $((|V|+|E|+4) \cdot|D|+(2 \cdot|\digamma|+1)$. $|E|+18) \cdot K+(|E|+2) \cdot K^{2}+3 \cdot|D|+1$.

\footnotetext{
${ }^{5}$ Here, since the coding overhead of R-NC is very small such that $\varepsilon \ll 1$ [29], we ignore it in the ILP formulation.
}

\section{Heuristic Algorithms}

In this section, we design several heuristics to perform MCRMSA that considers the physical impairments from both the transmission and light-splitting in EONs. Basically, in order to design an efficient MC-RMSA, we need to focus on improving the light-forest's spectral efficiency. Hence, the modulation selection for each light-tree becomes vital. However, the MTD relation in Eq. (1) makes the modulation selection relate to both the longest branch and the number of destinations in the light-tree. Specifically, a relatively high modulation-level may not be feasible for a large light-tree. Hence, we need to address the tradeoff between the modulation-level and the size of a light-tree carefully, and try to use the light-trees that can use relatively high modulation-level and cover many destinations.

\section{A. MC-RMSA using Set-Cover}

We first design an MC-RMSA algorithm that leverages the weighted set-cover problem [31]. For a multicast request $M R(s, D, B)$, the universe is the destination set $D$, the family $A$ represents the set of all the non-empty subsets of $D$. For instance, if $D=\left\{d_{1}, d_{2}\right\}$, then we have $A=$ $\left\{\left\{d_{1}\right\},\left\{d_{2}\right\},\left\{d_{1}, d_{2}\right\}\right\}$. We define $A_{m, n} \in A$ as the set of destinations within which any $n$ number of destinations can be covered by a light-tree with modulation-level $m$ according to the MTD relation. In the light-forest for $M R$, each lighttree has two key parameters, i.e., the number of destinations $n$ and the modulation-level $m$, which affect its spectrum consumption significantly. First of all, the more destinations that can be covered by the light-tree, the less light-trees will be needed by the light-forest (i.e., less bandwidth-variable transponders (BV-Ts)), and thus by increasing $n$, we can reduce the operational cost. On the other hand, the higher the modulation-level is, the more spectrum efficient the light-tree is, and hence by increasing $m$, we can reduce the total spectrum consumption of $M R$. Therefore, we define the weight of $A_{m, n}$ as $\frac{\beta}{n}+\frac{\delta}{m}$, where $\beta$ and $\delta$ are the positive constants to adjust the contributions of $n$ and $m$. Then, the MC-RMSA is transformed into the weighted set-cover problem that finds the minimum-weighted cover (i.e., a subset of $A$ ) whose elements have their union equal $D$.

In our algorithm, we first calculate all the shortest paths from source node $s$ to each destination node $d \in D$, denoted as $p_{s, d}$. Based on the MTD relation $S_{m, n}$ and the length of $p_{s, d}$, we can obtain the potential destination sets $\left\{A_{m, n}\right\}$. However, a set $A_{m, n}$ might be invalid, if the destinations in it are less than $n$. We remove these invalid sets. For the set $A_{m, n}$ that have more than $n$ destinations, we convert it to multiple sets by choosing $n$ destinations from it each time according to the distance between source and destinations, and when the remaining destinations are less than $n$, we just ignore them. After obtaining the updated destination sets, we find the minimum-weighted cover for them and use it to set up the light-forest for $M R$.

Algorithm 1 shows the detailed procedure of the MC-RMSA using set-cover (SC). Lines 1-8 are for the initialization, where we set the light-forest $\mathcal{T}$ and each set $A_{m, n}$ as empty and select the destinations to form a series of potential destination 
sets $\left\{A_{m, n}\right\}$. The for-loop that covers Lines 9-29 updates $\left\{A_{m, n}\right\}$ and the corresponding light-trees. We check whether a potential $A_{m, n}$ is valid with Lines 14-16. The for-loop covering Lines 18-27 calculates the light-trees based on a specific $A_{m, n}$. Specifically, we select $n$ destinations in $A_{m, n}$ each time, which are currently farthest from $s$, and then use a heuristic [32] to build a delay-constrained Steiner tree to cover $s$ and the destinations in $A_{m, n}$ while satisfying the maximum branch length $S_{m, n}$. The light-trees are then inserted into the light-forest $\mathcal{T}$, as shown in Line 22. Then, if $\mathcal{T}$ cannot cover all the destinations in $D$, Lines 30-31 mark the $M R$ as blocked. Otherwise, Lines 32-39 try to perform first-fit spectrum assignment [33] for all the light-trees in $\mathcal{T}$ and check whether $M R$ can be successfully served.

In Algorithm 1, since we can pre-calculate all the shortest paths between each node pair in the topology, the time complexity for checking whether the length of $p_{s, d}$ is within $S_{m, n}$ is $O(|V|)$, the complexity of constructing the delayconstrained Steiner tree is $O\left(|D|^{3}+|D| \cdot|V|\right)$ according to [32], in the worst case, the procedure will be performed $M \cdot|D|$. The complexity of assigning FS' to the light-forest is $O\left(|D| \cdot|V|^{2} \cdot|\digamma|\right)$. Hence, the time complexity of Algorithm 1 is $O\left(M \cdot|D|^{4}+M \cdot|D|^{2} \cdot|V|+|D| \cdot|V|^{2} \cdot|\digamma|\right)$.

\section{B. MC-RMSA using Set-Cover and Layered Auxiliary Graphs}

The second MC-RMSA heuristic leverages the good performance of the layered auxiliary graph (LAG) based approach that we proposed in [22]. Basically, the LAG approach can realize integrated multicast routing and spectrum assignment. Here, we combine SC with the LAG approach and propose the SC-LAG algorithm for MC-RMSA that considers the MTD relation. We define a threshold $m_{0}$, which is specific to a given topology $G(V, E)$, to divide the modulation-levels into two categories: 1) high modulation-levels $\left(m>m_{0}\right)$ and 2) low modulation-levels ( $\left.m \leq m_{0}\right)$. Here, for $M R(s, D, B)$, the meanings of $D$ and $A$ are the same as those in Subsection IV-A. Then, for an element in $A$ with the destination set that can be served with a high modulation-level according to the MTD relation, we still apply $\mathrm{SC}$ in $G(V, E)$ to serve the destinations, since SC uses relatively few FS' in total. However, if the destination set has to use a low modulationlevel, we apply the LAG approach and use SC in each LAG to avoid generating excessive spectrum fragmentation.

The details of the SC-LAG algorithm are given in Algorithm 2. In Lines 1-7, we run Algorithm 1 to find and serve all the light-trees that need high modulation-levels $\left(m>m_{0}\right)$. If there are still some destination(s) that have not be covered, Lines 839 try to serve them with the SC-LAG approach. The for-loop that covers Lines 10-20 builds the LAGs according to the spectrum usage in the network, and selects the destinations to form set $A_{m, n}^{i}$, which means that any $n$ destinations in $A_{m, n}^{i}$ can be served in the $i$-th LAG $G^{i}\left(V^{i}, E^{i}\right)$ with modulation-level $m$ according to the MTD relation. Specifically, to construct the $i$-th $G^{i}\left(V^{i}, E^{i}\right)$, we make $V^{i}=V$, check the spectrum usage in $G(V, E)$, and insert a link $e$ in $G^{i}\left(V^{i}, E^{i}\right)$, if starting from the $i$-th FS, there are $\left\lceil\frac{B}{C \cdot m}\right\rceil$ available contiguous FS' on $e$ in $G(V, E)$. Hence, if we can obtain a light-tree from $s$ to

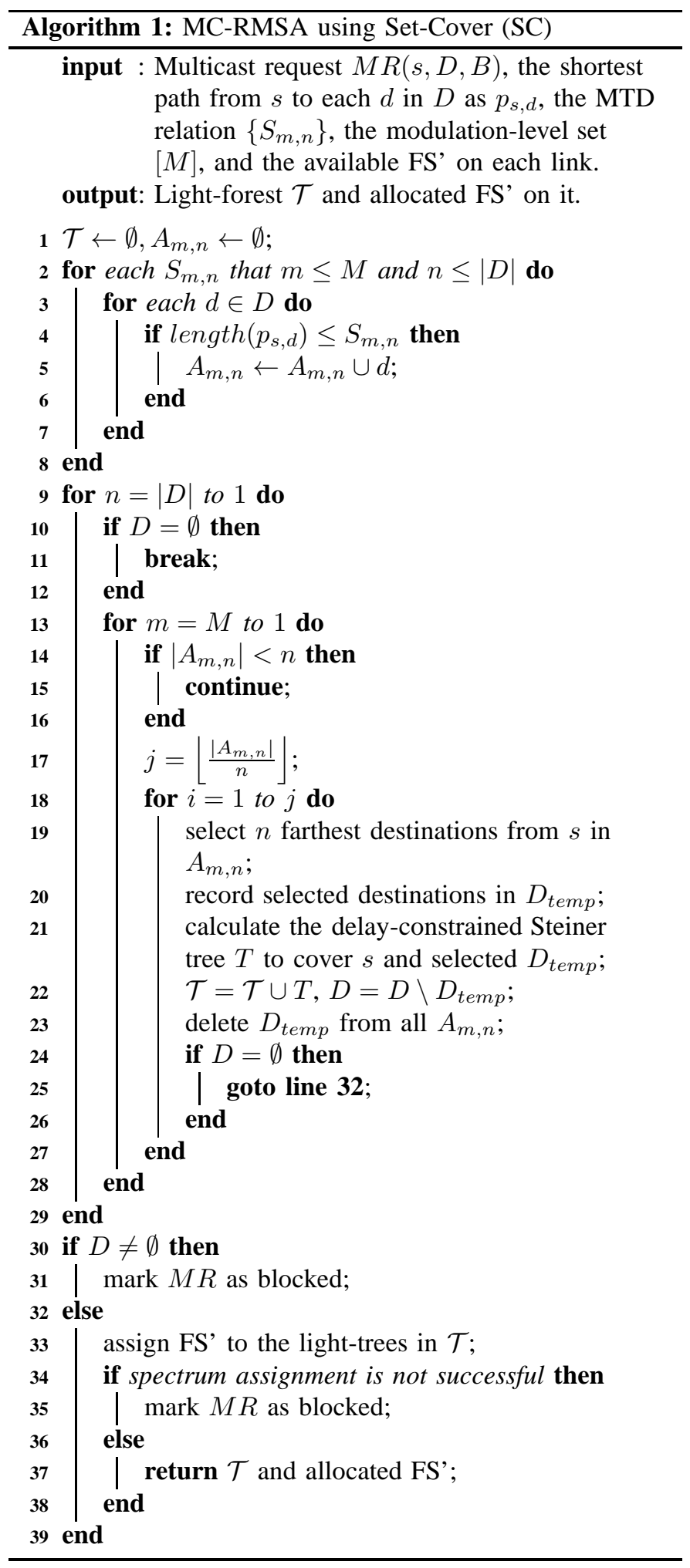




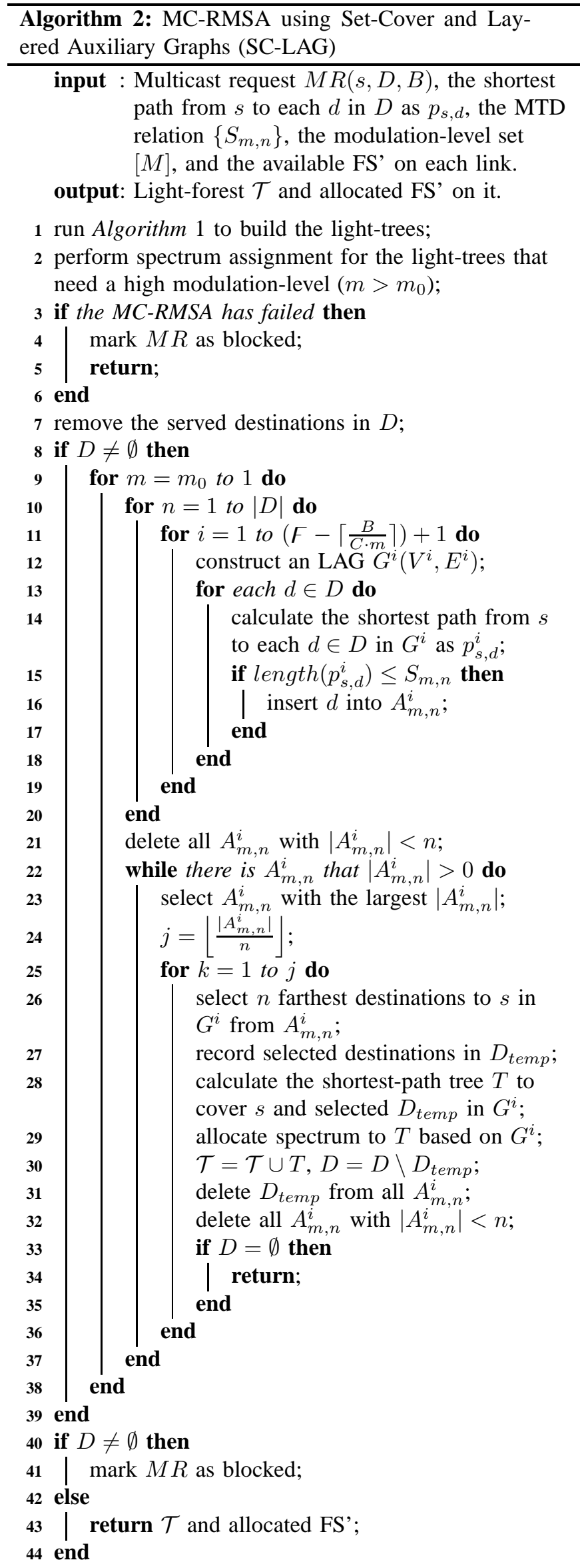

certain destinations in $G^{i}\left(V^{i}, E^{i}\right)$, those destinations can be served with the light-tree, using the $i$-th to $\left(i+\left\lceil\frac{B}{C \cdot m}\right\rceil-1\right)$ th FS' in $G(V, E)$. With all the $\left\{A_{m, n}^{i}\right\}$, the while-loop covering Lines 22-37 tries to serve the remaining destinations by building the largest feasible light-tree in the LAGs with the highest modulation-level each time. Finally, if certain destinations still have not been served, Lines 40-41 mark $M R$ as blocked, otherwise, the algorithm returns the light-forest $\mathcal{T}$ and allocated FS' on it for $M R$.

The time complexity of the Dijkstra's algorithm is $O(|E|+$ $|V| \cdot \log |V|)$ if we use the Fibonacci-heap data structure according to [34]. And the complexity of calculating the shortest-path tree is $O(|D| \cdot|V|)$ according to [35], if we know the shortest path from the source to each destination. And the complexity of deleting destinations from all the $\left\{A_{m, n}^{i}\right\}$ is $O(|\digamma| \cdot|D| \cdot M)$. Thus, the time complexity of the LAG part is $O(M \cdot(|\digamma| \cdot(|E|+|V| \log |V|)+|D| \cdot(|V|+|\digamma| \cdot|D|)))$. Finally, the time complexity of Algorithm 2 is $O\left(M \cdot|D|^{4}+\right.$ $M \cdot|D|^{2} \cdot|V|+|D| \cdot|V|^{2} \cdot|\digamma|+M \cdot(|\digamma| \cdot(|E|+|V| \cdot \log |V|)+$ $|D| \cdot(|V|+|\digamma| \cdot|D|)))$.

\section{MC-RMSA with R-NC using Set-Cover and Layered Aux- iliary Graphs}

Note that both SC and SC-LAG do not consider R-NC. Actually, we can easily extend SC-LAG and make it support the scheme that splits the traffic to certain destination(s) into multiple sub-streams and sends them over multiple light-trees, when $M R$ cannot be served due to lack of spectrum resources. More specifically, in Line 11 of Algorithm 2, we can replace $B$ with $B_{g}$ (i.e., the spectrum-splitting granularity) and build the LAGs accordingly. Also, before finishing the MC-RMSA, we need to make sure that all the destinations in $D$ can receive enough encoded bandwidth to recover the original data. This MC-RMSA heuristic is referred to as SC-LAG-R-NC.

\section{Performance Evaluation}

\section{A. Static Network Planning}

Since the heuristics in Section IV are designed for dynamic network provisioning, we make minor modifications in SCLAG-R-NC and SC-LAG to make them suitable for static network planning. Specifically, in SC-LAG-R-NC, the R-NC with light-forest is applied when a multicast request cannot be provisioned due to the lack of spectrum resources, which however, would not be an issue in static network planning. Hence, we modify this trigger condition to when serving a multicast request would increase the maximum index of the used FS' (MIUFS) in the network. Similarly, SC-LAG is also modified accordingly.

We evaluate the performance of the ILP model and heuristics in static network planning with the six-node topology shown in Fig. 5, considering both the cases with and without R-NC. All the simulations run on a computer with $3.40 \mathrm{GHz}$ Intel Core i3 CPU and 4 GB RAM and we use Lingo v11.0 [36] to solve the ILP. We assume that in the EON, an FS provides a capacity of $C=12.5 \mathrm{~Gb} / \mathrm{s}$ when using BPSK as the modulation format, and we choose two modulationlevels as $m=1$ (BPSK) and $m=3$ (8-QAM) to limit 
TABLE I

Simulation Results for Static Network Planning

\begin{tabular}{|c|c|c|c|c|c|c|c|c|c|c|}
\hline \multirow{2}{*}{ \# of Request } & \multicolumn{2}{|c|}{ ILP } & \multicolumn{2}{|c|}{ ILP-R-NC } & \multicolumn{2}{|c|}{$\mathrm{SC}$} & \multicolumn{2}{|c|}{ SC-LAG } & \multicolumn{2}{|c|}{ SC-LAG-R-NC } \\
\hline & MIUFS & $\begin{array}{l}\text { Running } \\
\text { Time (s) }\end{array}$ & MIUFS & $\begin{array}{l}\text { Running } \\
\text { Time (s) }\end{array}$ & MIUFS & $\begin{array}{l}\text { Running } \\
\text { Time (s) }\end{array}$ & MIUFS & $\begin{array}{l}\text { Running } \\
\text { Time (s) }\end{array}$ & MIUFS & $\begin{array}{l}\text { Running } \\
\text { Time (s) }\end{array}$ \\
\hline 5 & 8.4 & 63.368 & 7.2 & 1140.687 & 12.2 & 0.028 & 10.4 & 0.076 & 10.4 & 0.087 \\
\hline 10 & 15.4 & 161.119 & 12.6 & 1106.446 & 18.8 & 0.041 & 17.2 & 0.147 & 16.8 & 0.173 \\
\hline 20 & 24.6 & 956.641 & 20.6 & 3123.407 & 31.2 & 0.080 & 31.2 & 0.275 & 28.6 & 0.313 \\
\hline 35 & 39.0 & 1948.963 & 36.0 & 8638.648 & 52.6 & 0.135 & 47.4 & 0.507 & 46.6 & 0.580 \\
\hline 50 & 53.0 & 3134.702 & 50.0 & 9204.175 & 71.0 & 0.167 & 68.0 & 0.741 & 64.8 & 0.882 \\
\hline
\end{tabular}

the computational complexity. For each $M R(s, D, B)$, the source $s$ and destinations $D$ are randomly chosen, while $B$ is uniformly distributed within $[25,75] \mathrm{Gb} / \mathrm{s}$. The size of $D$ is set as 2 or 3 randomly, and the maximum number of light-trees in a light-forest is $K=3$.

Table I shows the simulation results on MIUFS in the network after serving all the multicast requests and the total computation time. In order to obtain each data point, we run the simulation 5 times and average out the results. Firstly, we discuss the performance difference between ILP and ILPR-NC. We observe that ILP-R-NC obtains smaller results on MIUFS than ILP but its computation time is also longer, and the difference between the results on MIUFS is not significant. There are two factors that limit the performance of ILP-RNC. One is that due to its high time complexity, we limit the maximum number of light-trees that can be included in a lightforest as $K=3$, which may make ILP-R-NC provide suboptimal solutions since the case with R-NC usually requires more light-trees to serve a multicast request. The other is that the six-node topology is too small, which also restricts the performance gap between ILP and ILP-R-NC. Secondly, we analyze the differences among the three heuristics. We observe that SC-LAG-R-NC provides the best performance on MIUFS since it can make network spectrum utilization more compact. While the performance of SC is the worst, since it considers the routing and spectrum assignment of a request separately. We also notice that SC-LAG and SC-LAG-R-NC have the same performance on MIUFS when the number of requests is 5. The reason is that the R-NC with light-forest is seldom applied for such a case in SC-LAG-R-NC. We can also see that for the heuristics, the trend on total running time is in the opposite direction of the performance on MIUFS.

Finally, it can be seen that the ILPs has better performance on MIUFS than the heuristics, but they also consume significantly longer computation time. Therefore, it is not practical to use the ILPs in large-scale networks and/or a dynamic network scenario that require real-time service provisioning decisions, considering the complexity and scalability. Thus, we will only discuss the time-efficient heuristics in the performance evaluation for dynamic network provisioning in the next subsection.

\section{B. EONs Provisioning With Dynamic Multicast Traffic}

In this subsection, we perform simulations with the two topologies shown in Figs. 6 and 7 for dynamic network provisioning. We consider that four modulation formats, BPSK,

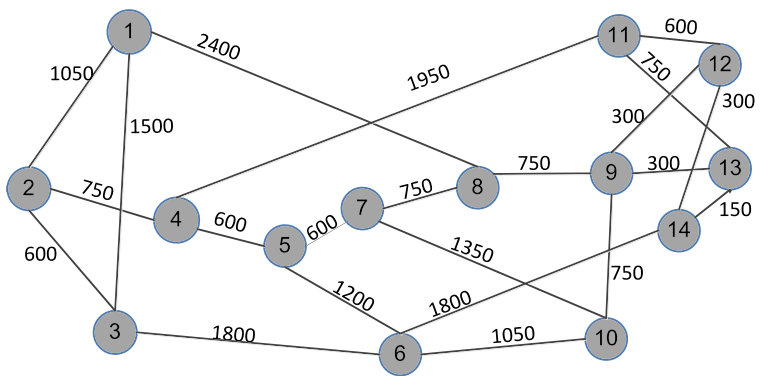

Fig. 6. NSFNET topology with fiber lengths in kilometers marked on links.

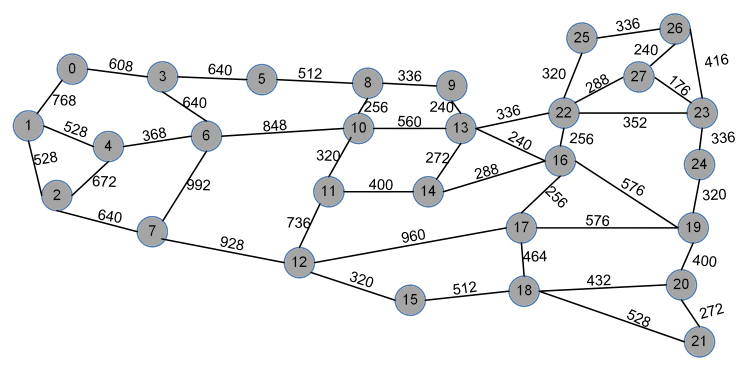

Fig. 7. US Backbone topology with fiber lengths in kilometers marked on links.

QPSK, 8-QAM and 16-QAM can be used in the EON. Each fiber link can accommodate $358 \mathrm{FS}$ ' (i.e., $\digamma=358$ ) that each has a capacity of $C=12.5 \mathrm{~Gb} / \mathrm{s}$ when using BPSK. The source and destinations are selected randomly from the topology, and for each traffic load, we simulate 10,000 requests. The capacity requirements of the multicast requests are uniformly distributed within $[50,100] \mathrm{Gb} / \mathrm{s}$, and the average number of destinations in the requests is 4 . We generate the requests according to the Poisson traffic model with $\lambda$ as the average arrival rate and $\frac{1}{\mu}$ as the average holding time. Then, the traffic load of multicast requests can be quantified with $\frac{\lambda}{\mu}$ in Erlangs. For SC-LAG and SC-LAG-R-NC, we set $m_{0}=1$ and $m_{0}=2$ for the NSFNET and US Backbone topologies in Figs. 6 and 7, respectively. This is because the average link lengths in NSFNET and US Backbone are $968.18 \mathrm{~km}$ and $466.49 \mathrm{~km}$, respectively. For SC-LAG-R-NC, we set the spectrum-splitting granularity as $B_{g}=\max \left(\left\lceil\frac{B}{4 \cdot C}\right\rceil, 1\right) \cdot C$.

Figs. 8(a) and 9(a) show the simulation results on blocking probability. It can be seen that in both topologies, SC provides the highest blocking probability. It performs worse than LAGbased approaches since LAG-based approaches achieve inte- 


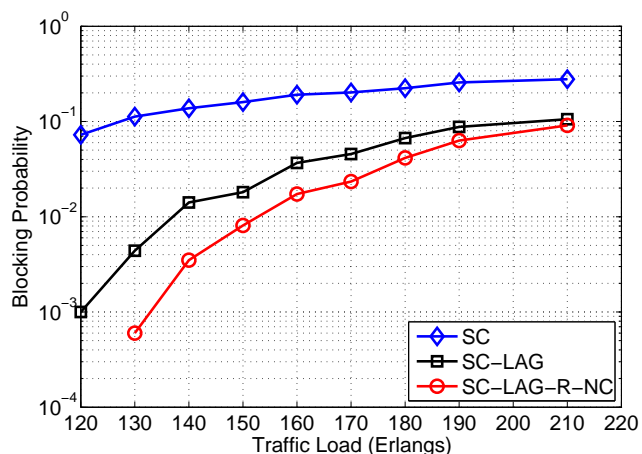

(a) Results on blocking probability.

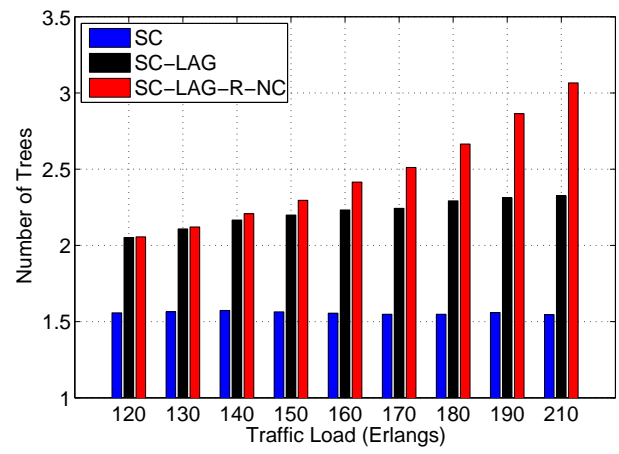

(b) Results on average number of light-trees per multicast request.

Fig. 8. Results from simulations with the NSFNET topology.

grated multicast routing and spectrum assignment and can alleviate spectrum fragmentation during the dynamic operation. Among the three heuristics, SC-LAG-R-NC performs the best. This is because the R-NC scheme with spectrum-splitting in SC-LAG-R-NC can leverage multiple sub-streams to provision relatively large traffic demands and arrange the light-trees well by using the LAG approach.

Nevertheless, even though the LAG-based approaches (SCLAG and SC-LAG-R-NC) can improve the blocking performance of the network compared with $\mathrm{SC}$, they require more light-trees per request than SC as illustrated in Figs. 8(b) and 9(b). This means that they may need more BV-Ts, which results in higher operational costs. Therefore, to provision the multicast requests, we have a tradeoff between the blocking performance and operational cost. It is also interesting to notice that the results on the average number of light-trees per request from SC and SC-LAG stay almost unchanged when the traffic load increases, but those from SC-LAG-RNC show noticeable increase when the traffic load is higher than 150 and 180 Erlangs in the NSFNET and US Backbone topologies, respectively. This is because when the traffic load is higher, SC-LAG-R-NC invokes the spectrum-splitting with $\mathrm{R}-\mathrm{NC}$ more frequently to serve more requests in the network.

\section{CONCLUSION}

This paper investigated the MC-RMSA schemes that consider the physical impairments from both the transmission and light-splitting in EONs, and proposed to serve each multicast

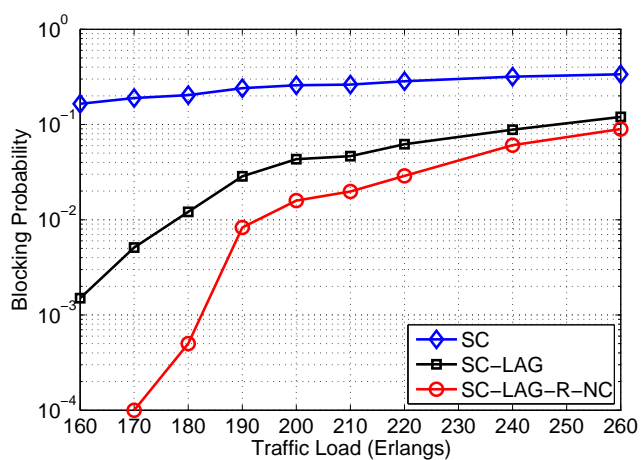

(a) Results on blocking probability.

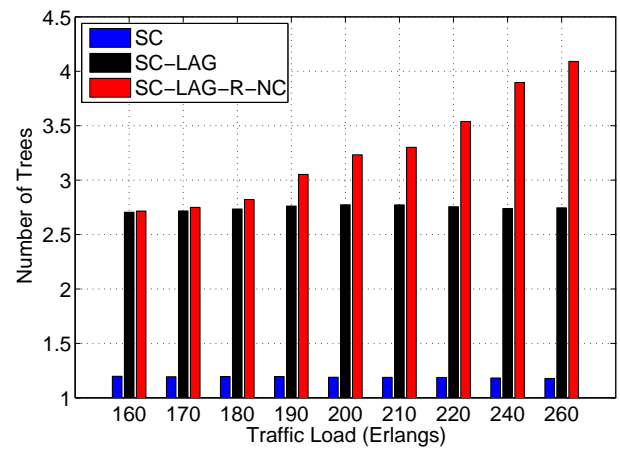

(b) Results on average number of light-trees per multicast request.

Fig. 9. Results from simulations with the US Backbone topology.

request with a light-forest that consists of one or more lighttrees. In order to further improve the spectral efficiency and compensate for the latency differences among the light-trees, we used the rateless network coding (R-NC) in the multicast system. An ILP model was first formulated to tackle the problem of static network planning. Then, we leveraged the setcover problem and utilized layered auxiliary graphs to design time-efficient heuristics. The simulation results showed that the MC-RMSA using light-forest with R-NC could effectively improve the performance of all-optical multicast in EONs.

\section{ACKNOWLEDGMENTS}

This work was supported in part by the NCET program under Project NCET-11-0884, the NSFC Project 61371117, the Fundamental Research Funds for the Central Universities (WK2100060010), Natural Science Research Project for Universities in Anhui (KJ2014ZD38), and the Strategic Priority Research Program of the CAS (XDA06011202).

\section{REFERENCES}

[1] W. Shieh, X. Yi, and Y. Tang, "Transmission experiment of multi-gigabit coherent optical OFDM systems over 1000km SSMF fibre," Electron. Lett., vol. 43, pp. 183-184, Feb. 2007.

[2] J. Armstrong, "OFDM for optical communications," J. Lightw. Technol., vol. 27, pp. 189-204, Feb. 2009.

[3] M. Jinno et al., "Distance-adaptive spectrum resource allocation in spectrum-sliced elastic optical path network [topics in optical communications]," IEEE Commun. Mag., vol. 48, pp. 138-145, Aug. 2010.

[4] O. Gerstel, M. Jinno, A. Lord, and S. Yoo, "Elastic optical networking: a new dawn for the optical layer?" IEEE Commun. Mag., vol. 50, pp. S12-S20, Feb. 2012. 
[5] K. Christodoulopoulos, I. Tomkos, and E. Varvarigos, "Elastic bandwidth allocation in flexible OFDM-based optical networks," J. Lightw. Technol., vol. 29, pp. 1354-1366, May 2011.

[6] L. Gong, X. Zhou, L. Wei, and Z. Zhu, "A two-population based evolutionary approach for optimizing routing, modulation and spectrum assignments (RMSA) in O-OFDM networks," IEEE Commun. Lett., vol. 16, pp. 1520-1523, Sept. 2012.

[7] Y. Sone et al., "Routing and spectrum assignment algorithm maximizes spectrum utilization in optical networks," in Proc. of ECOC 2011, p. Mo.1.K.3, Sept. 2011.

[8] Y. Wang, X. Cao, and Y. Pan, "A study of the routing and spectrum allocation in spectrum-sliced elastic optical path networks," in Proc. of INFOCOM 2011, pp. 1503-1511, Apr. 2011.

[9] M. Klinkowski, K. Walkowiak, and M. Jaworski, "Off-line algorithms for routing, modulation level, and spectrum assignment in elastic optical networks," in Proc. of ICTON 2011, pp. 1-6, Jun. 2011.

[10] X. Wan, N. Hua, and X. Zheng, "Dynamic routing and spectrum assignment in spectrum-flexible transparent optical networks," J. Opt. Commun. Netw., vol. 4, pp. 603-613, Aug. 2012.

[11] Z. Zhu, W. Lu, L. Zhang, and N. Ansari, "Dynamic service provisioning in elastic optical networks with hybrid single-/multi-path routing," $J$. Lightw. Technol., vol. 31, pp. 15-22, Jan. 2013.

[12] L. Sahasrabuddhe and B. Mukherjee, "Light trees: optical multicasting for improved performance in wavelength routed networks," IEEE Commun. Mag., vol. 37, pp. 67-73, Feb. 1999.

[13] W. Hu and Q. Zeng, "Multicasting optical cross connects employing splitter-and-delivery switch," IEEE Photon. Technol. Lett., vol. 10, pp. 970-972, Jul. 1998.

[14] M. Ali and J. Deogun, "Cost-effective implementation of multicasting in wavelength-routed networks," J. Lightw. Technol., vol. 18, pp. 16281638, Dec. 2000.

[15] G. Rouskas, "Optical layer multicast: rationale, building blocks, and challenges," IEEE Netw., vol. 17, pp. 60-65, Jan. 2003.

[16] X. Zhang, J. Wei, and C. Qiao, "Constrained multicast routing in WDM networks with sparse light splitting," J. Lightw. Technol., vol. 18, pp. 1781-1790, Dec. 2000.

[17] N. Singhal, L. Sahasrabuddhe, and B. Mukherjee, "Optimal multicasting of multiple light-trees of different bandwidth granularities in a WDM mesh network with sparse splitting capabilities," IEEE/ACM Trans. Netw., vol. 14, pp. 1104-1117, Oct. 2006.

[18] J. Wang, X. Qi, and B. Chen, "Wavelength assignment for multicast in all-optical WDM networks with splitting constraints," IEEE/ACM Trans. Netw., vol. 14, pp. 169-182, Feb. 2006.

[19] C. Hsieh and W. Liao, "All-optical multicast routing in sparse splitting WDM networks," IEEE J. Sel. Areas Commun., vol. 25, pp. 51-62, Aug. 2007.

[20] F. Zhou, M. Molnar, B. Cousin, and C. Qiao, "Cost bounds and approximation ratios of multicast light-trees in WDM networks," J. Opt. Commun. Netw., vol. 3, pp. 323-334, Apr. 2011.

[21] Q. Wang and L. Chen, "Performance analysis of multicast traffic over spectrum elastic optical networks," in Proc. of OFC 2012, p. OTh3B.7, Mar. 2012.

[22] X. Liu, L. Gong, and Z. Zhu, "Design integrated RSA for multicast in elastic optical networks with a layered approach," in Proc. of GLOBECOM 2013, pp. 2346-2351, Dec. 2013.

[23] L. Gong et al., "Efficient resource allocation for all-optical multicasting over spectrum-sliced elastic optical networks," J. Opt. Commun. Netw., vol. 5, pp. 836-847, Aug. 2013.

[24] A. Shokrollahi, "Raptor codes," IEEE Trans. Inf. Theory, vol. 52, pp. 2551-2567, Jun. 2006.

[25] A. Bocoi et al., "Reach-dependent capacity in optical networks enabled by OFDM," in Prof. of OFC 2009, pp. 1-3, Mar. 2009.

[26] M. Zhang, C. You, H. Jiang, and Z. Zhu, "Dynamic and adaptive bandwidth defragmentation in spectrum-sliced elastic optical networks with time-varying traffic," J. Lightw. Technol., vol. 32, pp. 1014-1023, Mar. 2014.

[27] A. Pages, J. Perello, S. Spadaro, and J. Comellas, "Optimal route, spectrum, and modulation level assignment in split-spectrum-enabled dynamic elastic optical networks," J. Opt. Commun. Netw., vol. 6, pp. 114-126, Feb. 2014.

[28] W. Lu et al., "Dynamic multi-path service provisioning under differential delay constraint in elastic optical networks," IEEE Commun. Lett., vol. 17, pp. 158-161, Jan. 2013.

[29] M. Luby, T. Gasiba, T. Stockhammer, and Watson, "Reliable multimedia download delivery in cellular broadcast networks." IEEE Trans. Broadcast., vol. 53, pp. 236-246, Jan. 2007.
[30] F. Zhou et al., "Minimizing server throughput for low-delay live streaming in content delivery networks," in Proc. of NOSSDAV 2012, pp. 65-70, Jun. 2012.

[31] V. V. Vazirani, Approximation Algorithms, C. of Computing Georgia Institute of Technology, Ed. Springer-Verlag, May. 2001.

[32] V. Kompella, J. Pasquale, and G. Polyzos, "Multicast routing for multimedia communication," IEEE/ACM Trans. Netw., vol. 1, pp. 286292, Jun. 1993.

[33] X. Zhou, W. Lu, L. Gong, and Z. Zhu, "Dynamic RMSA in elastic optical networks with an adaptive genetic algorithm," in Proc. of GLOBECOM 2012, pp. 2912-2917, Dec. 2012.

[34] M. L. Fredman and R. E. Tarjan, "Fibonacci heaps and their uses in improved network optimization algorithms," J. ACM, vol. 34, pp. 596615, Jul. 1987.

[35] M. Brown and R. Tarjan, "A fast merging algorithm," J. ACM, vol. 26, pp. 211-226, Apr. 1979.

[36] Lingo 11.0. [Online]. Available: http://www.lindo.com/index.php? option=com_content $\&$ view $=$ article $\&$ id $=151$ 